\title{
Every Six Weeks
}

National Cancer Institute

\section{Source}

National Cancer Institute. Every Six Weeks. NCI Thesaurus. Code C89788.

To be done at regular intervals of every six weeks. 